\title{
Adjuvant tamoxifen for operable carcinoma of the breast: report of clinical trial by the Christie Hospital and Holt Radium Institute
}

\author{
GERALD RIBEIRO, M K PALMER
}

\begin{abstract}
A large controlled clinical trial with the admission of 1005 patients was carried out using tamoxifen as adjuvant treatment for women with operable carcinoma of the breast. Results were analysed for the first 906 evaluable patients randomised up to December 1981 .

After mastectomy premenopausal women were randomised to receive either an irradiation menopause or tamoxifen $20 \mathrm{mg}$ daily for one year. Postmenopausal women were randomised to receive either tamoxifen $20 \mathrm{mg}$ daily for one year or no systemic treatment (controls). Analysis at five years suggested that for premenopausal women there was no significant difference between an irradiation menopause and tamoxifen in terms of survival, local recurrence, or distant metastases. Tamoxifen had no appreciable side effects. For postmenopausal women there was a trend in favour of tamoxifen with regard to survival and incidence of distant metastases, and the difference became statistically significant for those patients with four or more positive axillary nodes.

If long term results of these studies show only an improved quality of remaining life with tamoxifen, then this drug could be an important contribution to adjuvant treatment.
\end{abstract}

\section{Introduction}

The concept of adjuvant endocrine treatment for operable carcinoma of the breast is not new. As far back as 1948 a controlled clinical trial ${ }^{1}$ was initiated at the Christie Hospital in which patients after radical mastectomy were randomised to

\footnotetext{
Christie Hospital and Holt Radium Institute, Withington, Manchester M20 9BX

GERALD RIBEIRO, FFR, FRCR, consultant radiotherapist and coordinator of trial

M K PALMER, PHD, principal statistician

Correspondence and requests for reprints to: Dr Gerald Ribeiro.
}

have either ovarian irradiation (artificial menopause) or no further treatment. Analyses of the trial at 10 and 15 years showed a statistically significant reduction in the incidence of local recurrence and distant metastases within the ovarian irradiation group compared with the controls. The overall survival of the treated group, however, was not significantly improved. The benefit of an artificial menopause appeared to be least for women under 40 .

Since the first report by Cole $e t a l^{2}$ in 1971, tamoxifen has been widely used for advanced breast carcinoma in premenopausal and postmenopausal women. ${ }^{3}$ The drug is remarkably well tolerated when given by mouth. The main mode of action is by competitive interference with oestradiol uptake by specific receptors at various sites. The two major metabolites do not show any oestrogenic or teratogenic properties. ${ }^{4}$ Hence it was thought that tamoxifen would be very acceptable to patients if used as adjuvant treatment.

\section{Patients and methods}

Women aged 35-70 years who were fit enough for mastectomy and had no medical contraindications to tamoxifen or induction of an artificial menopause were eligible for the trial. Most would have operable tumours with a diameter of $5 \mathrm{~cm}$ or less clinically and palpable or impalpable mobile nodes in the ipsilateral axilla (clinical stages I and II $; T_{1}, T_{2}, N_{0}, N_{1 a}$ or $N_{1}, M_{0}$ ). A smaller proportion of women with more advanced but still operable disease were also eligible (clinical stage III; $\mathrm{T}_{3}, \mathrm{~N}_{0}, \mathrm{~N}_{1 \mathrm{a}}$ or $\mathrm{N}_{1}, \mathrm{M}_{0}$ ). Before operation the following investigations were done: full blood count, biochemical profile, and radiography of chest, lumbar spine, and pelvis. Initially the radiographs were repeated every six months but after two years this was stopped and radiography done only when clinically indicated.

Operation-All patients needed a mastectomy as a minimum procedure. For the trial three groups were defined: (1) simple mastectomy with no axillary dissection; (2) simple mastectomy with axillary node sampling; (3) radical mastectomy of the Patey type or simple mastectomy with total axillary clearance.

Treatment allocation-After operation, and hence histological confirmation, the patient was referred to a radiotherapist from the Christie Hospital and allocated at random to one of the following treatments: premenopausal women were allocated either to have an artificial menopause induced by ovarian irradiation or to receive tamoxifen $10 \mathrm{mg}$ twice daily for one year, and postmenopausal women 
were allocated to receive either tamoxifen $10 \mathrm{mg}$ twice daily for one year or no further treatment: this last group served as controls.

Postoperative radiotherapy was not given to patients who had had a simple mastectomy only or total axillary clearance or to those with histologically negative nodes in the axilla. Radiotherapy was given to patients who had histologically positive nodes on sampling the axilla and to all those with clinical stage III tumours. Postoperative radiotherapy was administered by one of two standard techniques used at the Christie Hospital at the time. ${ }^{5}$

Artificial menopause-Patients were regarded as premenopausal if they were actively menstruating, were within two years of the natural menopause, or had previously had a hysterectomy only and were aged less than 55 . The artificial menopause was induced by $x$ rays, using megavoltage treatment. For women aged 40 or less a mid-pelvic dose of $15 \mathrm{~Gy}$ (1500 rads) was given in four consecutive treatments, using a parallel opposed pair of fields. Women over 40 received a single exposure to give a midplane dose of $5 \mathrm{~Gy}$ (500 rads).

Randomisation and statistics-For various reasons it was not possible to carry out randomisation by telephone, so sealed envelopes were prepared in random permuted blocks and held at the Christie Hospital and at 12 peripheral hospitals; a master list was held centrally. Only a small number of radiotherapists actually participated in the randomisation of patients entered into the trial, and no problems arose. A form was filled in for each patient by the chief coordinator based on the medical records. All data were stored centrally in a computer at the medical statistics department of the Christie Hospital. Survival curves were produced by life table methods and compared in the various subgroups. Significant differences between these curves were examined by using the log rank test. ${ }^{6}$ The survival curves included deaths from causes other than breast cancer.

Follow up-All patients were followed up at similar intervals at the radiotherapy clinics. For the first year they were seen every three months, then every six months. When patients had moved abroad follow up was continued by writing to the patient's doctor. Patients continuing to live in Britain have now been "flagged" with the Office of Population Censuses and Surveys, so that the Christie Hospital will be automatically notified on the death of the patient.

Hormone receptor assays-It would have been ideal to carry out this form of investigation within a hormone based adjuvant trial. At the time that the trial began, however, this investigation was, and still is, a research facility and not available throughout the north western region. The decision was therefore made to go ahead with the trial on the basis that other trials using tamoxifen with a similar type of surgery but starting at a later date would probably have hormone receptor assays incorporated within the protocol.

\section{Results}

From 1 November 1976 to 1 June 1982, when the trial closed, 1005 patients had been entered. The present paper is concerned with the 948 patients entered up to December 1981. Forty two of the 948 patients were excluded from the analysis owing to violations of protocol. In most cases the patients were too old, and in the remainder the clinical staging was wrong, a mastectomy had not been done, or extensive systemic treatment had been given just before randomisation. Most of these problems occurred in the first few months of the trial. Twenty patients were excluded from the tamoxifen group and 22 from the artificial menopause and control groups. Thus of the 948 patients, 906 were available for analysis, of whom 453 had been allocated to receive tamoxifen and 453 to undergo induction of an artificial menopause or be followed up only as postmenopausal controls. The mean age was 54 years in each group.

Four of the patients randomised to receive tamoxifen were not given the drug, two patients randomised to have an artificial menopause were given tamoxifen by mistake, and two patients had oophorectomies done instead of induction of artificial menopause. To avoid any slight bias that might otherwise have occurred in the analysis patients were considered to belong to the group to which they were randomly allocated irrespective of the treatment they in fact received.

Of the patients allocated to tamoxifen, $94 \%$ completed a year on the drug, $2 \%$ took the drug for a year in a reduced dose of $10 \mathrm{mg}$ daily, and $4 \%$ stopped the drug owing to side effects.

Based on clinical staging, the numbers of patients randomised were 612 with stage I disease, 192 with stage II, and 102 with stage III. Table I shows the treatments allocated after the three types of operation. The histological state was known in 630 cases $(70 \%)$.

The various treatment groups were analysed and found to be comparable for age, site of tumour, type of surgery, state of the nodes, and menstrual state.
TABLE I-Treatment allocated after the three types of operation. Distributio expressed as number (\%) of 906 patients analysed

\begin{tabular}{|c|c|c|c|c|}
\hline \multirow[b]{2}{*}{$\begin{array}{l}\text { Treatment allocated } \\
\text { after surgery }\end{array}$} & \multicolumn{3}{|c|}{ Operation performed } & \multirow[b]{2}{*}{ Total } \\
\hline & $\begin{array}{c}\text { Simple } \\
\text { mastectomy } \\
\text { only }\end{array}$ & $\begin{array}{l}\text { Simple } \\
\text { mastectomy } \\
\text { with node } \\
\text { sampling }\end{array}$ & $\begin{array}{c}\text { Radical } \\
\text { mastectomy }\end{array}$ & \\
\hline $\begin{array}{l}\text { Tamoxifen } \\
\text { Artificial menopause/control }\end{array}$ & $\begin{array}{l}139(31) \\
136(30)\end{array}$ & $\begin{array}{l}193(42) \\
200(44)\end{array}$ & $\begin{array}{l}121(27) \\
117(26)\end{array}$ & $\begin{array}{l}453(100) \\
453(100)\end{array}$ \\
\hline
\end{tabular}

\section{STATE AT TIME OF ANALYSIS}

Of the 453 patients allocated to tamoxifen, 403 were alive and $5 \overline{\overline{\hat{\sigma}}}$ were dead-47 from breast cancer and three from proved intercurren causes. In the artificial menopause/control groups 389 were alive an 8 64 were dead-60 from breast cancer and four from intercurreno disease.

\section{PREMENOPAUSAL PATIENTS}

A total of 354 patients were premenopausal, of whom 189 wer randomised to receive tamoxifen and 165 to have an artificial menoo pause. Figure 1 shows the survival curves for the two groups. Therg were 19 deaths in the tamoxifen group and 20 deaths in the artificiag menopause group $(\mathrm{p}=0 \cdot 59)$.

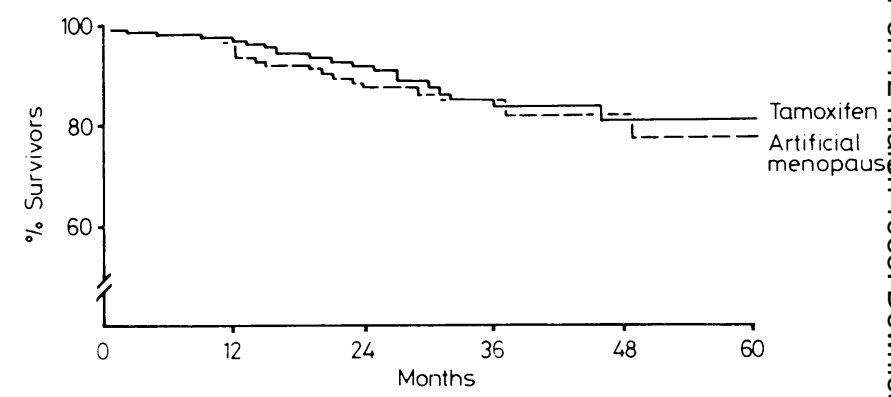

FIG 1-Survival of premenopausal women (354 patients).

In the subgroup with histologically negative nodes (pathologicall stage I) 104 patients were randomised. Only four of these died, two in the tamoxifen group and two in the artificial menopause group. The four year survival of the tamoxifen group was $92 \%$ versus $94 \%$ for the artificial menopause group. Neither adjuvant treatment was therefore apparently harmful to this group of patients.

Analysis of the 117 patients with histologically positive nodes in the axilla suggested a survival trend in favour of tamoxifen, but the difference was not statistically significant $(p=0 \cdot 22)$.

An artificial menopause was successfully induced in all but six of 161 patients who had ovarian irradiation. The six patients subsequentlo became menopausal after further irradiation to their ovaries. All this patients eventually developed hot flushes, and in those actively menstrus ating the periods disappeared. In $83(48 \%)$ of 172 menstruating patien given tamoxifen there was some effect on periods, 28 of them becoming irregular and 46 developing amenorrhoea. Periods returned to normat in most cases within one year of stopping the drug. Five patients developed menorrhagia, of whom two had a hysterectomy showing uterine fibroids, two had dilatation and curettage (one with normas curettings, the other with cystic hyperplasia), and one underwerf induction of an artificial menopause. The only other side effects were hot flushes in five patients and nausea in six. The hot flushes were not severe and stopped when tamoxifen was withdrawn after one yean Only two patients had to stop the drug because of severe nausea.

\section{POSTMENOPAUSAL PATIENTS}

Of the 552 patients who were postmenopausal, 264 were allocate to receive tamoxifen and 288 to serve as controls-that is, to receive. no adjuvant treatment and be followed up only. Figure 2 shows the survival of these patients. There were 31 deaths in the tamoxifer 
group compared with 44 among the controls. Overall, the differences were not statistically significant ( $p=0.21)$.

There were 177 postmenopausal patients with histologically negative nodes in the axilla. Only two patients in the tamoxifen group died compared with eight in the control group ( $p-0.09)$.

Analysis of the 212 patients with histologically positive nodes showed that at five years the control group had had 21 deaths and the tamoxifen group $23(p-0.62)$. Figure 3 shows the survival of a sub-

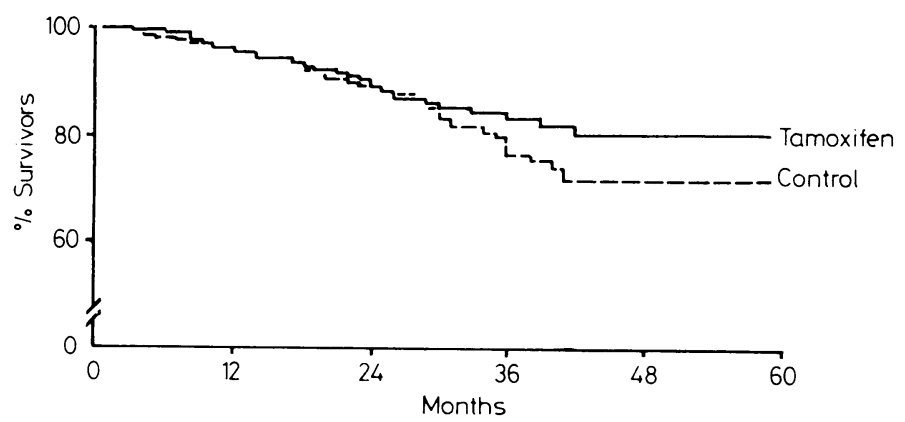

FIG 2-Survival of postmenopausal women (552 patients).

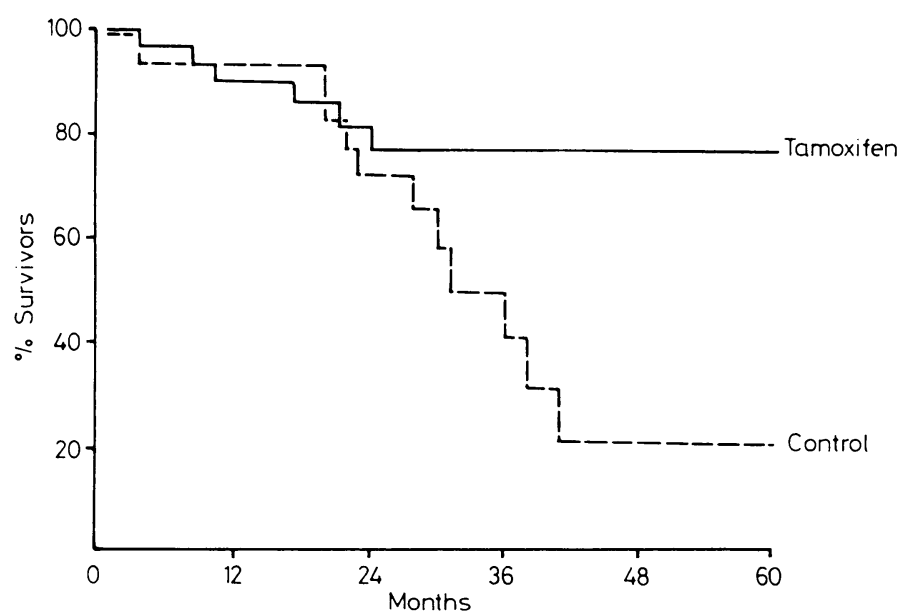

FIG 3-Survival of postmenopausal women with four or more diseased axillary nodes (67 patients).

group of 67 postmenopausal patients with four or more nodes showing histological evidence of disease. Only six deaths occurred in the tamoxifen group compared with 12 in the control group, the difference being statistically significant $(p=0 \cdot 03)$.

\section{LOCAL RECURRENCE}

Local recurrence was defined as recurrent disease in the operative flaps in the ipsilateral axilla, supraclavicular fossa, and internal mammary regions. A total of 98 patients developed local recurrences; of these, 46 were also found to have distant metastases, either simultaneously or subsequently, and 32 of these $46(70 \%)$ died. Of the remaining 52 patients with local recurrence only, seven died. The loal recurrence in $47 \%$ of the 98 patients was thus only a manifestation of much more widespread disease.

If allowance is made for postoperative radiotherapy, then at the time of analysis there was no statistically significant difference in the incidence of local recurrence between patients who had received tamoxifen and those in the artificial menopause/control groups.

\section{DISTANT METASTASES}

There was no significant difference in the incidence of distant metastases between patients in the tamoxifen group and those in the artificial menopause/control groups. Of the 146 patients who developed distant metastases, $38(26 \%)$ had lung metastases and $64(44 \%)$ had bone metastases at some time. These metastases were within the areas surveyed by the initial screening radiographs. Table II shows the proportion of positive $x$ ray findings in the first two years when radiography was done at six monthly intervals. With 777 examinations done at an average cost of $£ 30$ each it was not a cost effective screening procedure. It is debatable whether adding whole body isotope bone scans would have made an appreciable difference to the detection rate.

TABLE II-Proportions of patients with positive radiological findings in first two years

\begin{tabular}{lcccc}
\hline & \multicolumn{5}{c}{ Time (months) } \\
\cline { 2 - 5 } & 6 & 12 & 18 & 24 \\
\hline No screened & 290 & 263 & 122 & 102 \\
No (".) with positive result & $9(3)$ & $24(9)$ & $10(8)$ & $12(12)$ \\
\hline
\end{tabular}

\section{SYSTEMIC TREATMENT FOR RECURRENCE AND METASTASES}

While in theory it would have been ideal to have a crossover of treatments, in practice it does not always work out that way. In fact, most patients from the control group who developed further disease were treated with tamoxifen. Of 45 evaluable patients so treated, 14 had a complete or partial response. Seven patients underwent induction of an artificial menopause and 21 were treated with cytotoxic drugs. The remainder were given other forms of hormone treatment or received no treatment.

\section{Discussion}

It would be unwise to draw firm conclusions from these early results. Some points, however, merit further discussion.

For premenopausal women tamoxifen adjuvant treatment is apparently not inferior to an artificial menopause. Criticism has been levelled at the low dose ( $4.5 \mathrm{~Gy}$ ( $450 \mathrm{rads}$ ) on kilovoltage) used in Cole's original trial ${ }^{1}$ but, in fact, $90 \%$ of women aged 40 years and over had disappearance of their periods. The high rate of failure was in women aged under 40 , and for these women we increased the dose in the present series to $15 \mathrm{~Gy}$ (1500 rads) in four treatments on megavoltage with complete success. In the present series only $4 \%$ of women given $5 \mathrm{~Gy}$ (500 rads) required further treatment.

Only $30(17 \%)$ of 172 women treated with tamoxifen had permanent amenorrhoea. Not all women wish to have a permanent menopause inflicted on them, so that if the same therapeutic effect can be obtained with tamoxifen then it is a viable alternative. It may be argued that ovarian irradiation is cheaper but this has to be balanced against the quality of life offered to the individual patient.

For postmenopausal women overall survival did not significantly improve at five years, but a subgroup of women with four or more positive nodes in the axilla was defined as showing significant benefit from adjuvant tamoxifen.

If long term results of the present adjuvant chemotherapy trials show only a significant delay in the appearance of metastases with either modest or no improvement in survival, then the quality of life of the patient is of paramount importance. In the latter case, tamoxifen could make an important contribution to adjuvant treatment.

The obvious advantages would be ease of administration (there is now a $20 \mathrm{mg}$ tablet-Nolvadex D-available), lack of side effects, and the possibility that the drug could be given on a long term basis with less toxicity than one would expect from cytotoxic drugs.

Our thanks are due to Professor Michael Baum and Mr Alan Wilson, of the surgical unit at King's College Hospital, London, who carried out an external assessment of this trial four years after its 
inception. Our thanks are also due to Imperial Chemical Industries PLC, who kindly provided all the tamoxifen (Nolvadex) tablets for the duration of the trial, and, finally, to Miss M J Moores and Miss J L Parsons for typing the manuscript.

Surgeons who participated in the trial were: $M$ A Brennan, K G Buckler, M Crumplin, D G Cowley, H A Daniels (Retd), J Done, J Duthie, A J Farmer, R C Hartley, A Higham, N Hira, W Humphreys, J Kelly, J B Laine, W Lawson, W H Lonsdale, J P Lythgoe, J Main, Miss M T Morrell, W G Paley, J Russell (Retd), A F Robinson, I Sewell, W N Samarji, H F Smith, J S S Stewart, D Teasdale (Retd), L Turner, J B L Taylor, R S Todd, A Watson, J Whitehead, A W Williams (Retd), W Weatherston-Wilson (Retd), and J Yule.

Radiotherapists who participated were: E Allan, M P Cole (Retd), D P Deakin, M B Duthie, R Gibb (Retd), N K Gupta, R D Hunter, R D James, D Pearson, R S Pointon, G Read, G G Ribeiro, E Sherrah-Davies, R Sout, and M L Sutton.
References ${ }^{1}$ Cole MP. A clinical trial of an artificial menopause in carcinoma of the
breast. IN

${ }^{2}$ Cole MP, Jones CTA, Todd IDH. A new anti-oestrogenic agent in late breast cancer. An early clinical appraisal of ICI 46474. Br f Canceß $1971 ; 25: 270-5$.

3 Mouridsen H, Palshof T, Patterson J, Battersby L. Tamoxifen in advancedo breast cancer. Cancer Treat Rev 1978;5:131-41.

${ }^{4}$ Jordan VC, Dowse LJ. Tamoxifen as an anti-tumour agent: effect on oestrogen binding. J Endocrinol 1976;68:297-303.

${ }^{5}$ Lythgoe JP, Leck T, Swindell R. Manchester Regional Breast Study'. preliminary results. Lancet $1978 ; \mathrm{i}: 744-7$.

${ }^{6}$ Peto R, Peto J. Asymptotically efficient rank invariant test procedures? fournal of the Royal Statistical Society 1972;A135:185-207.

(Accepted 8 February 1983)

\title{
Effect of metformin on insulin receptor binding and glycaemic control in type II diabetes
}

\author{
J M LORD, S I WHITE, C J BAILEY, T W ATKINS, R F FLETCHER, K G TAYLOR
}

\begin{abstract}
To investigate the effect of metformin on insulin receptor binding and diabetic control, eight obese type II diabetic patients were studied before treatment, after one and four weeks of taking metformin ( $500 \mathrm{mg}$ thrice daily), and four weeks after withdrawal of the drug. After one and four weeks of treatment the number of erythrocyte insulin receptors had increased by $116 \%$ and $184 \%$ respectively. This was due almost entirely to an increase in the number of low affinity binding sites. The number of receptors was still raised four weeks after metformin had been withdrawn. Diabetic control as assessed by urinary glucose, glycosylated haemoglobin $\left(\mathbf{H b} A_{1}\right)$, and glucose tolerance values was significantly improved during metformin treatment, while plasma insulin concentrations were not altered.

These results indicate that metformin produces a rapid and protracted increase in low affinity insulin receptors in type II diabetes, associated with greater insulin sensitivity and improved diabetic control.
\end{abstract}

\section{Introduction}

Metformin has a place in the treatment of type II diabetic patients with normal hepatic and renal function. The drug may be appropriate in obese patients who show an inadequate

\footnotetext{
Department of Biological Sciences, University of Aston in Birmingham, Birmingham B4 7ET

J M LORD, BSC, research fellow

C J BAILEY, PHD, lecturer in physiology

$T$ W ATKINS, PHD, lecturer in biochemistry

Department of Diabetes and Endocrinology and Clinical Investigation Unit, Dudley Road Hospital, Birmingham B18 7QH

S I WHITE, MRCP, senior house officer (now registrar in dermatology, Royal Victoria Infirmary, Newcastle upon Tyne)

R F FLETCHER, FRCP, consultant physician

K G TAYLOR, MD, consultant physician
}

response to diet and in non-obese patients in whom a combination $\vec{v}$ of diet and sulphonylurea has failed to produce glycaemic control

Metformin reduces hyperglycaemia by increasing periphera glucose uptake and reducing hepatic gluconeogenesis. The effect of the drug is achieved without an increase in plasma insulin concentrations, suggesting that it potentiates the action of insulin. ${ }^{1}$ The first step in the cellular mechanism of insuling action is the binding of insulin to receptors in the plasms membrane. Metformin reportedly increases insulin binding to receptors in erythrocytes of normal subjects ${ }^{2}$ and to receptors inf cultured lymphocytes and cultured breast cancer cells. ${ }^{3} \mathrm{~W} \mathbb{Q}_{2}$ report the effect of metformin on erythrocyte insulin receptor binding in type II diabetes in relation to diabetic control.

\section{Patients and methods}

We studied eight obese patients with type II diabetes (mean $137.5 \pm$ SEM $10 \cdot 2 \%$ of ideal body weight). Five of the patients were postmenopausal women and three were men, and their mean age waš $61 \cdot 0+4.6$ years. The mean time since diagnosis of their disease was $4.0 \pm 0.8$ years. Each patient was asked to follow a fully structured energy controlled diet from four weeks before the study until itso completion. The diet was designed to maintain a constant body weight throughout. No patient had proteinuria or any biochemical evidences of impaired renal or hepatic function and none received any medication other than metformin. Each patient served as his or her own control: Tests were performed immediately before treatment, after one and four weeks of treatment with metformin (Glucophage, $500 \mathrm{mg}$ threq times a day), and four weeks after the metformin had been withdrawn.w

Patients presented at 0900 after a 12 hour fast. A 24 hour urine collection from the previous day was analysed for the glucose conceneo tration. ${ }^{4}$ Venous blood was collected for measurement of the percent $\frac{-}{-}$ age of glycosylated haemoglobin $\left(\mathrm{HbA}_{1}\right)^{5}$ and plasma concentrations of glucose, ${ }^{4}$ insulin, ${ }^{6}$ and metformin. ${ }^{7}$ A $50 \mathrm{~g}$ oral glucose tolerance testo was performed and blood samples for measurement of plasma glucose and insulin concentration taken at 0,30, 60, 90, and 120 minutes.

Erythrocyte insulin receptor binding was determined by the method of Gambhir et al. ${ }^{8}$ Data were analysed after Scatchard ${ }^{9}$ using a mode which assumes two classes of receptors with different but fixed affinities, one exhibiting high affinity and the other low affinity. ${ }^{10}$ Then apparent receptor affinity was taken as the concentration of native insulin required to reduce by $50 \%$ the specific cell bound fraction of ${ }^{125}$ I-insulin. ${ }^{11}$ The different interpretations of insulin receptor binding data have been reviewed elsewhere. ${ }^{12}$ 